\title{
LAS REDES DE ATENCIÓN A LA INFANCIA COMO RESPUESTA A LOS CAMBIOS EN EL MUNDO ACTUAL
}

\author{
Secanilla, E. \\ Universitat Oberta de Catalunya (UOC), Barcelona. \\ esecanilla@uoc.edu \\ esther.secanilla@gmail.com
}

Fecha de Recepción: 12 Febrero 2018

Fecha de Admisión: 10 Abril 2018

\section{RESUMEN}

La Psicología de la Educación ha de dar respuesta al mundo cambiante actual. La finalidad de esta investigación fue ofrecer herramientas que promovieran dichos cambios, a través del asesoramiento psicopedagógico. Se revisó y analizó el concepto de red social desde una perspectiva psicoeducativa adoptando un enfoque sistémico. Se analizaron las características de las redes de atención a la infancia iniciadas en algunas poblaciones. El estudio permitirá plantear los elementos para llevar a cabo el asesoramiento sobre el diseño e implementación de una red de atención a la infancia. Como prospectiva se plantea la posibilidad de realizar dicho asesoramiento mediante la intervención en la creación de diferentes redes de atención a la infancia y la adolescencia.

Palabras clave: asesoramiento psicopedagógico; redes de atención a la infancia; trabajo interdisciplinario

\footnotetext{
ABSTRACT

The networks of attention to the infancy like answer to the changes in the current world.

The Psychology of the Education must give response to the current changing world. The purpose of this investigation is to offer tools that promote those changes through the psychopedagogical advice. The concept of social network is reviewed and analysed from a psychologic and educative perspective adopting a systemic approach. The characteristics of the networks of attention to the childhood initiated in the populations to Catalonia are deeply analysed. The study will allow to pose the elements to carry out the advice on the design and implementation of a network of attention to the childhood. The future work is to achieve this advice through the intervention in the creation of different networks of attention to the childhood and the adolescence.

Keywords: psychopedagogical advice; networks of attention to the childhood; interdisciplinary work
} 


\section{LAS REDES DE ATENCIÓN A LA INFANCIA COMO RESPUESTA A LOS CAMBIOS EN EL MUNDO ACTUAL}

\section{ANTECEDENTES DE LA TEMÁTICA A TRATAR}

Nuestra sociedad está en un proceso de cambio constante, al igual que la educación, y la Psicología de la Educación debiera dar respuesta a dichos cambios. En nuestro entorno hay infinidad de formas de concretar los servicios a la infancia y a sus familias. Profesionales de diversos sectores e instituciones del ámbito formal y no formal coinciden en ofrecer recursos, soporte y atención a la infancia. Así pues, se hace necesario ofrecer un trabajo en equipo, un trabajo interdisciplinario, en red que permita ir construyendo modelos prácticos y teóricos. Trabajar en red resulta muy enriquecedora para los diversos estamentos implicados (Del Carmen, 2001: 161).

Hablar de red social en ciencias de la educación y psicología implica abordar otras disciplinas, como la física o la matemática, la tecnología o la antropología, hasta llegar a la psicología social y a la terapia familiar. Fue el etnógrafo y antropólogo John A.Barnes (1990, pp.67-97), en sus estudios sobre las relaciones interindividuales en una aldea de pescadores en Noruega quien incorporó el concepto de redes sociales analizando los relaciones que se producían entre los habitantes de la población. Elisabeth Bott (1968) habla de los tipos de roles conyugales comparándolas a la red de la familia. Sluzki (1996) aborda terapia de red asimilándola a la terapia familiar. Moreno (1975) estudia sobre las relaciones entre los individuos y el grupo. Barudy (1998) aporta una infinidad de recursos a partir del modelo de redes en los servicios de protección a la infancia.

\section{OBJETIVOS DE LA INVESTIGACIÓN}

La finalidad de esta investigación fue ofrecer herramientas que promovieran dichos los cambios de la sociedad, a través del asesoramiento psicopedagógico. Previamente se estudiaron las características de las redes de atención a la infancia iniciadas en cuatro poblaciones de los alrededores de Barcelona, analizando a fondo el desarrollo de una de ellas, para llegar a proponer un asesoramiento sobre el diseño e implementación de una red de atención a la infancia (Secanilla, 2016b), que se señalará en este estudio.

\section{PARTICIPANTES}

La Red de Infancia de Sant Feliu de Llobregat fue creada por el Ayuntamiento como soporte técnico y social a las familias de la población. Se inició en 1997, fundamentándose en la coordinación entre equipos de profesionales del ámbito público y privado de diferentes sectores como el educativo, la sanidad, los servicios sociales, la policía local, la biblioteca, asociaciones, etc. Su misión era mejorar y hacer disminuir las desigualdades en la atención a los niños y adolescentes de la población, mediante el incremento de la cantidad y calidad del soporte a las familias en su tarea de crianza y educación, con el apoyo de los servicios que formaban la Red. Se trataba de un proyecto intersectorial y comunitario que consiguió ser hasta 2004 un elemento clave para la comunicación entre los profesionales, la ciudadanía y para dar a conocer su experiencia a otros municipios.

La red de infancia de Castelldefels abre sus puertas en 2009, comenzando a conocerse como la casa de los Niños. Es la denominación de los centros educativos municipales para la primera infancia y sus familias, que responde a un modelo de centro integral de calidad, disponiendo de espacios adecuados que ofrecen actividades educativas para los niños y para sus familias. Se centra en la diversidad de necesidades de los niños por lo que ofrece un amplio abanico de horarios que la familia puede escoger en función de sus necesidades. Actualmente se está ampliando a nivel de red interna para ofrecer más recursos a niños más mayores.

En la ciudad de Terrassa los centros y servicios para la infancia están gestionados por el Patronato Municipal de Educación, organismo autónomo del Ayuntamiento. Hay diversas escuelas para la primera infancia que trabajan en forma de Red. 
En la Ciudad de Cerdanyola del Vallès se estudió en profundidad el funcionamiento en red de una de las escuelas de primera infancia -Gespa- creada en 1974 hasta 2015. Dicha escuela formaba parte del Programa de Escuelas Verdes impulsado por el Departamento de Educación y de Medio ambiente de Catalunya. La escuela planteaba su trabajo pedagógico a partir de la construcción de la personalidad del niño y el conocimiento de su entorno a través de la experimentación y manipulación de diversos materiales, así como con la interrelación que se establece con su grupo de iguales y con los adultos de referencia. Se consideraba un espacio de interacción entre las familias y los niños, dinamizándose por los profesionales del centro y del entorno.

\section{METODOLOGÍA}

Para analizar la calidad psicosocioeducativa de la oferta de redes y servicios se diseñaron instrumentos como un sistema de indicadores que permitiera evaluar la atención psicosocioeducativa a la infancia recibida en los centros y servicios de las poblaciones (Secanilla, 2016), así como cuestionarios y encuestas para analizar el funcionamiento de las redes, donde se recogía información a partir del análisis de los objetivos y características de las redes y del análisis de aspectos organizativos y metodológicos de la implementación, seguimiento y evaluación de las Redes de Atención a la Infancia. Se realizaron entrevistas a técnicos y un exhaustivo estudio documental de actas, registros y memorias de las poblaciones.

Tabla 1. Estructura de las encuestas

\begin{tabular}{|c|c|c|c|}
\hline DIMENSIONES & & CATEGRÍAS & SUBCATEGORÍAS \\
\hline $\begin{array}{l}\text { 1.OBJETIVOS } \\
\text { CARACTERÍSTICAS } \\
\text { RED }\end{array}$ & DE $\quad \begin{array}{r}\text { LA } \\
\end{array}$ & $\begin{array}{l}\text { 1.1. Objetivos de la red } \\
\text { 1.2. Características de la red }\end{array}$ & $\begin{array}{l}\text { 1.1.1. Objetivos prioritarios } \\
\text { 1.1.2. Otros objetivos } \\
\text { 1.2.1. Funcionamiento } \\
\text { 1.2.2. Implicación de los } \\
\text { profesionales y de las familias } \\
\text { 1.2.3. Beneficios de la Red para los } \\
\text { profesionales y para las familias }\end{array}$ \\
\hline $\begin{array}{l}\text { 2.ASPECTOS } \\
\text { ORGANIZATIVOS } \\
\text { RED }\end{array}$ & DE LA & $\begin{array}{l}\text { 2.1. Organización general de la } \\
\text { Red }\end{array}$ & $\begin{array}{l}\text { Organigrama, recursos humanos y } \\
\text { materiales, circuitos establecidos } \\
\text { para trasmitir informaciones, } \\
\text { informaciones interred, } \\
\text { informaciones a los centros y a los } \\
\text { servicios, informaciones a las } \\
\text { familias, coordinación. } \\
\text { Objetivos de los grupos de trabajo, } \\
\text { áreas de trabajo de los } \\
\text { profesionales que participan, } \\
\text { frecuencia de las reuniones, } \\
\text { organización interna, coordinación } \\
\text { entre los grupos de trabajo, } \\
\text { participación de las familias y los } \\
\text { niños, material de trabajo, } \\
\text { evaluación del trabajo realizado, } \\
\text { publicación de las evaluaciones, } \\
\text { valoración de los técnicos } \\
\text { coordinadores. }\end{array}$ \\
\hline
\end{tabular}

\section{RESULTADOS ALCANZADOS}

Se obtuvieron una serie de elementos prioritarios a partir del análisis de las entrevistas. Se consideró que los objetivos prioritarios de las redes analizadas eran dos, a saber: 


\section{LAS REDES DE ATENCIÓN A LA INFANCIA COMO RESPUESTA A LOS CAMBIOS EN EL MUNDO ACTUAL}

Atender a los niños y a sus familias ofreciéndoles ayuda en el proceso de desarrollo y aprendizaje de sus hijos (Intervención),

Partir de un verdadero trabajo de coordinación entre diversos profesionales de diferentes ámbitos, como eje básico de trabajo (Coordinación).

La intervención que las redes realizan es una intervención directa, las especificaciones se centran en cada grupo de trabajo, a partir de los cuales se decide la manera de abordar cada intervención directa. Además, las redes proponen potenciar los grupos de trabajo intersectorial y el ámbito social de las redes, así pues, mejorar la coordinación entre los equipamientos. La coordinación es el eje vertebrador del trabajo en red.

Otros objetivos que se proponen las redes de atención a la infancia son el de prevención, detección, planificación del trabajo y evaluación.

A partir del análisis de las encuestas y de los resultados obtenidos, se procedió a establecer un modelo teórico de asesoramiento para la creación de redes de atención a la infancia. Asesorar para implementar una red de infancia y adolescencia en una población conlleva a adoptar un modelo que tenga en cuenta el contexto, pero también las interacciones que se producen entre los agentes, es decir, un modelo de asesoramiento psicopedagógico participativo (Carretero, Pujolás, Serra, 2002). Este modelo apunta a que sus actores, asesorados y asesor, creen una interacción de interdependencia, y la toma de decisiones sea consensuada y compartida entre todos. Como línea conductora, se considera necesario incorporar elementos éticos en la intervención, como la honestidad, plantear los límites de la intervención, evitar generar expectativas que superen las posibilidades técnicas de actuación, conseguir el beneficio de los implicados, ser prudente y mantener la confidencialidad, entre otras (Bonals y De Diego, 2001).

Por otra parte, se procedió a proponer un diseño para la creación de redes de atención a la infancia (Secanilla, 2016b). En las líneas estratégicas de asesoramiento se incluyen aspectos como los niveles de maduración del equipo, el liderazgo, el compromiso compartido, la creación de grupos de trabajo, entre otros. Se debe contar con una estructura organizativa que permita tener en cuenta sus características estructurales como por ejemplo el tamaño, la distribución y el tipo de funciones que se establezcan (Sluzki, 1996). Los actores que intervienen deben estar interrelacionados, de forma que la población sienta que hay un entramado global que acoge a cada profesional (Rovere, 1999), por lo que se proponen diferentes niveles en el proceso de construcción de la red, además de unas fases generales y la necesaria evaluación. Como instrumentos de análisis utilizados se proponen documentos de trabajo, actas, que entre los participantes elaboran conjuntamente.

Crear redes a partir de estos elementos de asesoramiento y evaluación se convierte en una respuesta para mejorar la calidad de los recursos de la población, además de mejorar la coordinación entre profesionales y ahorrar duplicidades innecesarias.

\section{DISCUSIÓN}

A partir del análisis de las respuestas obtenidas se observó una serie de aspectos clave para que funcionen las redes de atención a la infancia en cualquier población: Es necesario una iniciativa política, un compromiso por parte de los ayuntamientos de las poblaciones, además de un compromiso compartido por las diversas administraciones. Es necesario también que las tareas de los profesionales que se implican en las redes estén contempladas en su horario laboral y no que se prevea como un voluntariado. Sería conveniente que los profesionales de un mismo centro o servicio fuesen alternando su intervención en la red de la población para posibilitar la participación de todos. Es necesario ofrecer u reconocimiento a la tarea realizada por los profesionales que forman parte de las redes. Además, los servicios de cada comunidad debería involucrarse de una manera espontánea en el trabajo en red. Otro aspecto a tener en cuenta es que es necesaria una estructura organi- 
zativa, funcional y personal para poder llevar a cabo un trabajo en red, por todo lo cual se ha propuesta una serie de líneas estratégicas para llevar a cabo un trabajo en red que sea realmente compartido y útil para la población donde se implante. En estos momentos de cambio son necesarias propuestas de esta índole para afrontar las necesidades del tiempo presente, no obstante, se requiere un nivel organizativo importante y un buen asesoramiento para llevar a cabo un trabajo real de red. Los retos en un futuro vienen determinados por estos aspectos.

\section{CONCLUSIONES}

En esta investigación, se ha presentado el estudio de diferentes modelos de trabajo en red, a partir de los cuales se ha diseñado una propuesta de asesoramiento para la creación de redes de atención a la infancia y sus familias. Se ha podido valorar los elementos necesarios para que sea posible diseñar una red de atención y evaluar sus resultados.

No podemos obviar los beneficios que comporta un trabajo interdisciplinario dentro de un trabajo en red como modelo, beneficios para los mismos profesionales (soporte mutuo entre técnicos, tener espacios de reflexión, formación conjunta, traspaso de informaciones entre servicios, comprender a las familias y el proceso de atención a la infancia desde diversas perspectivas y en cada momento social), beneficios para mejorar la calidad de los centros y servicios que atienden a las personas, beneficios para las familias, para los niños y para la población en general. Por este motivo es importante impulsar el trabajo en red y debería contemplarse como uno de los objetivos en las poblaciones, eso sí, con un buen asesoramiento psicopedagógico para su creación y evaluación.

\section{REFERENCIAS BIBLIOGRÁFICAS}

Azam, M., De Federico, A. (2014). Sociología del arte y análisis de redes sociales [versión electrónica]. REDES. Revista hispana para el análisis de redes sociales. 25(2), 1-22.

Barnes, J.A. (1990). Models and interpretations: selected essays. Cambridge: Cambridge University Press.

Barudy, J. (1998). El dolor invisible de la infancia. Barcelona: Paidós.

Bertalanffy, L.V. (1976). Teoría general de los sistemas: fundamentos, desarrollo, aplicaciones. México: Fondo de Cultura Económica.

Boissevain, J. Mitchell, J. Clyde (1973). Network Analysis: Studies in Human Interaction. Paris: Mounton.

Bonals, J., De Diego, J. (2001). Ética y estética de una profesión en desarrollo. En Monereo, C. y Solé, I. (Coord.). El asesoramiento psicopedagógico: una perspectiva profesional y constructivista.(pp.501-515). Madrid: Alianza Editorial.

Börzel, T. (1997). Policy networks: a new for European governance? Badia Fiesolana, San Domenico $(\mathrm{FI})$ : European University Institute, Florence. Robert Shuman Centre.

Bott, E. (1968). Family and social network: roles, norms, and external relationships in urban families. London: Tavistock.

Bronfenbrenner, U. (1987). La ecología del desarrollo humano. Barcelona: ediciones Paidós.

Carretero, M.R., Pujolàs, P., Serra, R. (2002). Un altre assessorament per a l'escola. L'assessorament psicopedagògic des d'una perspectiva comunitària. Barcelona: La Galera, SA Editorial.

Casals, A., De Vicente, I., Garriga, R., Tabueña, C.M. (2005). Intervenció social en I'atenció sociosanitària i residencial. Barcelona: UAB.

Castells, M. (1997). La era de la información: Economía, sociedad y cultura. Volumen I: La sociedad red. Madrid: Alianza Editorial. 


\section{LAS REDES DE ATENCIÓN A LA INFANCIA COMO RESPUESTA A LOS CAMBIOS EN EL MUNDO ACTUAL}

Del Carmen LI. (2001). El trabajo en equipo: aspecto básico para la innovación en los centros. En Monereo, C. y Solé, I. (Coord.). El asesoramiento psicopedagógico: una perspectiva profesional y constructivista. (pp.153-161) Madrid: Alianza Editorial.

Gay, E.I. (2005). Xarxes locals d'infància. Reflexions i una experiència. [versión electrónica]. De Prop. Revista de política educativa local. Textos. 13, 1-19.

Johnson, David W. et al (1999). El aprendizaje cooperativo en el aula. Buenos Aires; Barcelona: Paidós.

Johnson, David W., Johnson, Frank P. (1996). Joining together: group theroy and group skills. Boston, Mass: Allyn and Bacon.

Johnson, David W., Johnson, Roger T. (1989). Cooperation and competition: theory and reserche. Minnesota: Interaction Book Company.

Maturana, R. (1995). Desde la biología a la psicología. Santiago de Chile: Editorial universitaria.

Maturana, R. (1997). Emociones y lenguaje en educación y política. Santiago de Chile: Dolmen ediciones.

Maturana, R. y Varela, G. (1984). El árbol del conocimiento. Las bases biológicas del entendimiento humano. Santiago de Chile: Lumen-editorial universitaria.

Maturana, R. y Varela, G. (1994). De máquinas y seres vivos. Autopoiesis: la organización de lo vivo. Santiago de Chile: Editorial universitaria Lumen.

Merelo, J.J. (2005). Redes sociales: una introducción. Revista hispana para el análisis de redes sociales. [versión electrónica]. Artículo introductorio a la Ciencia de las redes. Taller. http://revista-redes.rediris.es/webredes/talleres/redes.pdf

Mitchell, J. Clyde (1987). Cities, Society, and social perception. Oxford: Clarendon Press.

Moreno, J.L. (1975). Psicoterapia de grupo y psicodrama. Méjico: Fondo de Cultura Económica.

Rodríguez-Roca, J.; Honrubia, M.L., Guardia, J. (2005). La cultura de la evaluación de programas: un elemento fundamental para afrontar los retos de las políticas sociales. Revista de Servicios Sociales y política sociales. Núm. 50, 55-70.

Rovere, M. (1993). Planificación estratégica de recursos humanos en salud. [versión electrónica]. Whashington, D.C. EUA: Organización panamericana de la salud.

Rovere, M. (1999). Redes en salud; un nuevo paradigma para el abordaje de las organizaciones y la comunidad. [versión electrónica]. Rosario, República Argentina: Editorial Secretaría de la salud pública/AMR, Instituto Lazarte.

Secanilla, E. (2016). La atención psioeducativa en la primera infancia. Evaluación de centros, servicios y programas. Editorial Uoc.

Secanilla, E. (2016b). Redes de atención a la infancia. Una propuesta de asesoramiento psicopedagógico en su diseño e implementación. Pedagogia i Treball Social Revista de Ciències Socials aplicades. Vol.5, Num. 2: 28-51

Sennett, R. (2003). El respeto. Sobre la dignidad del hombre en un mundo de desigualdad. Barcelona: Anagrama.

Sluzki, Carlos E. (1996). La red social: frontera de la práctica sistémica. Barcelona: Gedisa.

Traveset, M. (2007). La pedagogía sistémica. Fundamentos y práctica. Barcelona: Editorial Graó.

Ubieto, J.R. (2007). Modelos de trabajo en red.[versión electrónica].Revista de educaciónsocial.Núm.35. 